\title{
CORRESPONDENCE
}

\section{Iran's scientists condemn instances of plagiarism}

The Iran chapter of the Academy of Sciences for the Developing World, speaking for the country's academic community, deplores the recent cases of alleged plagiarism by Iranian scientists (see Nature 462, 704-705: 2009).

Iran's scientific community is largely free of such unethical behaviour. The calibre of its scientific output is reflected by the substantial growth in recent years in its share of research articles published in high-quality, peer-reviewed international journals.

Several factors account for this improvement in Iran's research output, including sustained and generous government support for science, a swelling of the ranks of young researchers and increasing international collaboration.

The Internet has facilitated communication with our colleagues elsewhere - but the availability of journals on the Internet has also made plagiarism easier. This widely acknowledged problem affects the scientific community worldwide. Iran, sadly, is no exception, and the country's science community is overhauling its practices to counter this scourge.

But circumstances more specific to Iran are also conducive to the spread of plagiarism. Iranian culture places an excessive emphasis on the value of academic credentials, both for advancement in official professions and in social standing. In particular, Iran's political class has an unusual affinity for possessing academic distinctions, as exemplified by the fact that a university degree is a prerequisite for election to parliament. A higher degree is also considered an important qualification for holding other government offices. As a result, the Iranian political class, across the political and ideological spectrum, accounts for a disproportionate share of academic fraud.

Farhad Ardalan, Hessamaddin Arfaei, Reza Mansouri Sharif University of Technology and the Institute for Research in Fundamental Sciences (IPM), Tehran, Iran Mahdi Balalimood Mashhad University of Medical Sciences, Mashhad, Iran Dariush Farhud, Reza Malekzadeh Medical University of Tehran, Iran Habib Firouzabadi, Keramatollah Izadpanah-Jahromi, Afsaneh Safavi, Shiraz University, Shiraz, Iran Ali Kaveh Iran University of Science and Technology, Tehran, Iran Farrokh Saidi, Abbas Shafiee Shahid Beheshti Medical University, Tehran, Iran

Yousef Sobouti Institute for Advanced Studies in Basic Sciences, Zanjan, Iran

\section{Opening dialogue between the recent and the long ago}

Douglas Erwin's call for palaeontologists to move towards a better understanding of diversity (Nature 462, 282-283; 2009) should be extended. Palaeoecologists should move beyond purely descriptive objectives and towards a better understanding of ecosystem evolution.

The worlds of palaeoecologists and ecologists are very much apart, and dialogue between the two remains muted. (One exception is the Quaternary, in which several key researchers have had a strong ecological background.)

Palaeoecologists working across geological time should, we suggest, familiarize themselves with the paradigms being debated in the ecological literature, and seek ways with which they could be examined in the fossil record. Palaeoecological research has to rely on uniformitarian assumptions using modern ecological analogues. Similarly, ecologists could be more sensitive to the limitations of data collection from the historical record, and could help frame modern studies to complement palaeoecological hypothesis testing.

The way modern and fossil ecosystems are described will necessarily be different, but researchers should strive to see whether the rules described for modern ecosystems hold over geological time. After all, the fossil record provides the only way to study changes in ecosystems over more than centennial timescales, along with samples of nonanalogous ('extinct') ecosystems and habitats.

As first steps, palaeoecologists could publish in leading ecological journals and participate in ecological conferences.

Julien Louys, Laura C. Bishop, David

M. Wilkinson Research Centre in Evolutionary Anthropology and Palaeoecology, School of Natural Sciences and Psychology, Liverpool John Moores University, Liverpool L3 3AF, UK e-mail: j.louys@ljmu.ac.uk

\section{UK defence group's structure could limit its usefulness}

You describe a new UK Ministry of Defence programme, in a News story (Nature 462, 151; 2009), as being modelled on its US counterpart, the JASONs: this is an independent group of scientific advisers with high-level security clearance who have consulted for the US government on technical problems since the cold war. As a member of the JASONs for more than a decade, I note that several organizational aspects are crucially different.

First, the British government intends to finance research in the laboratories of members of their advisory group in order to follow up on ideas that they generate. Second, most members won't have security clearance. Third, members will not be selfselected, but will be appointed by the government. Fourth, the membership will be rotated frequently according to topic.
In my opinion, these differences detract from the usefulness of the UK enterprise. Lack of clearance discourages members from bringing up relevant issues, and will hobble security-related work generally. Controversial conclusions of reports could be dismissed by government officials with the all-too-familiar refrain: "You wouldn't advise that if you knew what we know."

Also, the group stands to forfeit independence because the government will select scientists according to its own criteria, which tend to include political expediency in addition to competence. They risk losing credibility within the scientific community for the same reason. When members are funded to pursue projects at their home institutions, it raises conflictof-interest issues. And rotating the membership will prevent the organization from ever developing a true 'corporate memory'.

As an organization, the JASONs have earned credibility on a wide range of security-related topics over the years. The long-standing working relationships forged among the JASON members rank among its most significant strengths. The lack of direct professional ties to government sponsors fosters impartiality. The collective experience gained from working on so many different kinds of problem, combined with the individual credentials of its scientifically diverse membership, make the JASONs one of the few government advisory groups that can plausibly be called independent.

Steven M. Block Departments of Biology and Applied Physics, Stanford University, Stanford, California 94305-5020, USA e-mail: sblock@stanford.edu

Contributions to this page may be sent to correspondence@nature. com. Please see the Guide to Authors at go.nature.com/ cMCHno. We also welcome comments at Nautilus (http:// blogs.nature.com/nautilus). 